\title{
Structure Extraction Using Relative Total Variation
}

\author{
Ch. Parameshwara Rao ${ }^{1}$, Ch. Madhuri ${ }^{2}$, G.Shyam Sundhar ${ }^{3}$, J. Anil Chandra ${ }^{4}$ \\ V. Nancharaiah ${ }^{5}$ \\ ${ }^{1,2,3,4,5}$ Department of Electronics and Communication Engineering, Lendi Institute Of Engineering and \\ Technology, Affiliated to JNTUK, Vizianagaram.
}

\begin{abstract}
It is used to separate background from main structures in images, drawings, and paintaings .images consists of main structure and the texture. our new relative total variation method which will be presented in this paper is used to eliminate the complete texture based on different properties between the texture and structure. .By performing the no of iterations we can get the effective structure. This is also used to extracting the boundries of the image by varying the values of the different parameters. We propose new algorithm which capture the essential difference of these two types of visual forms, and develop an efficient optimization system to extract main structures. The new variation measures are validated on millions of sample patches. Our approach finds a number of new applications to manipulate, render, and reuse the immense number of "structure with texture" images and drawings that were traditionally difficult to be edited properly.,
\end{abstract}

Keywords: texture, structure, smoothing, total variation, relative total variation,

\section{Introduction}

Image consists of texture plus structure. In our daily life lakhs of such pictures and drawings can be found in the internet.. Texture elements forms the meaningful structures. We call them as "structure+ texture" images. A lot of effort is needed to obtaining the structures by a computer.Manual manipulation is needed in all photo editing softwares. A few methods are available however, theycannot effectively separate texture from the main structures because both of them could receive similar penalties during optimization. Recent edgepreserving image editing tools aim to solve the similar penaities probleme and they are not optimal. More analysis and comparisons will be provided. We present a simple and effective method based on novel local variation measures to eliminate texture removal. We found that our new relative total variation, which will be presented in this paper is used to eliminate the complete texture based on different properties.

Our method makes large number of existing "structure+ texture" images reusable in editing and rendering. It can be used in several applications including structural edge detection, vectorization, seamless cloning, and structure-only image composition.This method makes the results are less sensitive to errors compare to other methods. As our method assumes neither the specific type of texture nor the latent main structure arrangement, it cannot distinguish between texture and structure that are similar in scales or are close with respect to the new variation measures. The method performs best for lighting that is not very complex and images without strong perspective distortion when user interaction is not involved. While this is not an issue for imges. such as well-lit paintings, drawings and mosaics on which the paper focuses, this canbe more problematic written as with the anisotropic expression in $2 \mathrm{D}$.

\section{Background Process}

Texture usually refers to surface patterns that are similar in appearance and local statistics synthesis can produce a large seamless texture map from small examples. For near-regular textures, spatial relationship is used to detect and analyze regularity enabling imagetexture separation in de-fencing These methods count on the symmetry and regularity of texture and require prior pattern knowledge. Image analogy needs examples and may have difficulty removing texture when details are complex and irregular.

Representative structure-texture decomposition methods that do not require extensive texture information are those enforcing the total variation (TV) regularizer to preserve large-scale edges is most favorable with unknown texture pattern.The TV-L2 model simply uses a quadratic penalty to enforce structural similarity between the input and output, expressed as argmin where $I$ is the input, which could be the luminance (or $\log$ luminance) channel and $p$ indexes 2D pixels. $S$ is the resulting structure image. The data term $\_\boldsymbol{S p}-\boldsymbol{I} \boldsymbol{p} \_\boldsymbol{2}$ is to make the extracted structures similar to those in the input image.

$$
\arg \min _{S} \sum_{p}\left\{\frac{1}{2 \lambda}\left(S_{p}-I_{p}\right)^{2}+\left|(\nabla S)_{p}\right|\right\},
$$

Fig.1. Background Process Equation 


\section{Approach}

We do not assume or manually determine the type of textures, as thepatterns could vary a lot in different examples. Our method contains a general pixel-wise windowed total variation measure, written as where $q$ belongs to $R(p)$, the rectangular region centered at pixel $p$.

$$
\begin{aligned}
\mathscr{D}_{x}(p) & =\sum_{q \in R(p)} g_{p, q} \cdot\left|\left(\partial_{x} S\right)_{q}\right|, \\
\mathscr{D}_{y}(p) & =\sum_{q \in R(p)} g_{p, q} \cdot\left|\left(\partial_{y} S\right)_{q}\right|, \\
\mathscr{L}_{x}(p) & =\left|\sum_{q \in R(p)} g_{p, q} \cdot\left(\partial_{x} S\right)_{q}\right|, \\
\mathscr{L}_{y}(p) & =\left|\sum_{q \in R(p)} g_{p, q} \cdot\left(\partial_{y} S\right)_{q}\right| .
\end{aligned}
$$

Eq 3(a)

$L$ captures the overall spatial variation. Different from the expression in Eq. 3(a), it does not incorporate the modulus. So the sum of $\partial S$ depends on whether the gradients in a window are coincident or not, in terms of their directions, because $\partial S$ for one pixel could be either positive or negative. Key Observation There is an important finding on $L$ that guides our system design - that is, the resulting $L$ in a window that only contains texture is generally smaller than that in a window also including structural edges. An intuitive explanation is that a major edge in a local window contributes more similar-direction gradientsthan textures with complex patterns. We show a $L$ map in where the texture, albeit visually salient, produces smaller $L$ values than the main structures. It is not a special example. We will show in Eq 3 and 3(a) that this finding is actually acquired statisticallyfrom many data. To further enhance the contrast between texture and structure, especiallyfor visually salient regions, we combine $L$ with $D$ to form an even Relative Total Variation (RTV) is simple and yet very effective to make main structures stand out, thanks to the characteristics of $D$ and $L$.

$$
g_{p, q} \propto \exp \left(-\frac{\left(x_{p}-x_{q}\right)^{2}+\left(y_{p}-y_{q}\right)^{2}}{2 \sigma^{2}}\right),
$$

Eq 3(b)

Normalization using windowed inherent variation $L$ is similar to circular and spherical statistics (CSS), where the norm of the sum of unit vectors is used to normalize spherical mean and variance. One term in CSS evaluates the concentration of vectors. Our inherent variation shares similarities with these spherical metrics, which yields small responses when local gradients scatter, corresponding to textures. It differs from CSS on incorporating a windowed total variation and working in concert with a data fidelity term.

\section{Strategy And Comparison}

To verify the effectiveness of the RTV measure, we build a dataset, which contains millions of patches along with manually created labels. In the first place, we collect 200 "structure+texture" images and ask five student helpers to draw strokes snapping to important Structure Extraction from Texture via Relative Total Variation structure edges. The remaining pixels are treated as not containing meaningful changes. So each image has a corresponding stroke map. A few test images and corresponding stroke maps are shown in the blueframe Based on them, we wrote a program to randomly draw structure and texture patches respectively, all with size $29 \times 29$. Structure patches contain labeled strokes while the texture patches do not.By normalizing the measures and varying the classification threshold in [0,1], we plot precision-recall curves in For comparison, we also evaluate widowed total variation and windowed $L 0.8$ regularizer that approximates the sparse prior in the WLS method. Our relative total variation has a clear superiority over other alternatives. Difference to texture classification Note that our final goal is not texture/structure classification, but instead another challenging task, i.e., texture removal from different "structure+texture" images. 


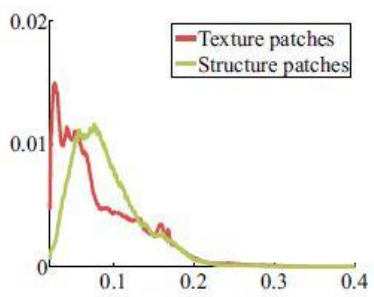

(a) $\mathscr{D}_{x}+\mathscr{D}_{y}$

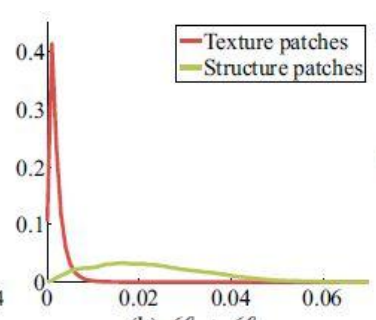

(b) $\mathscr{L}_{x}+\mathscr{L}_{y}$

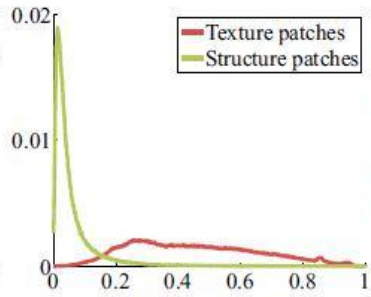

(c) RTV measure

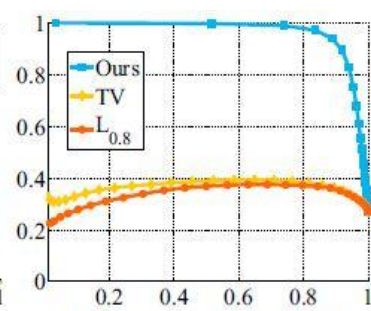

(d) Precision-recall curve

Fig 4(a) Comparison with Previous Methods

\section{Enhancing Of Image Processing Using Algorithm}

The objective function in Eq. 3(b) is non-convex. Its solution thus cannot be obtained trivially. We propose an efficient solver based on the knowledge that an objective function with the penalty of a quadratic measure can be optimized linearly

Our approach decomposes the RTV measure into a non-linear term and a quadratic term. The advantage is that the problem with the nonlinear part, intriguingly, can be transformed to solving a series of linear equation systems, in a way similar to iterative re-weighted least squares. We first discuss the $x$-direction measure. The $y$-direction term can be dealt with similarly. We expand the penalty as By re-organizing the terms and grouping elements that contain

\section{Algorithm 1 Structure Extraction from Texture}

1 : input: image $I$, scale parameter $\sigma$, strength parameter $\lambda$

2: initialization: $t=0, S 0 \leftarrow I$

3: for $\mathrm{t}=0: 2$ do

4: compute weights $w$ and $u$ ), and

5: solve the linear system in

6: end for

7: output: structure image $S$

\section{Experimental Results}

Upon Performing Several Simulations by MATLAB Tools.The obtained Results are as Follows

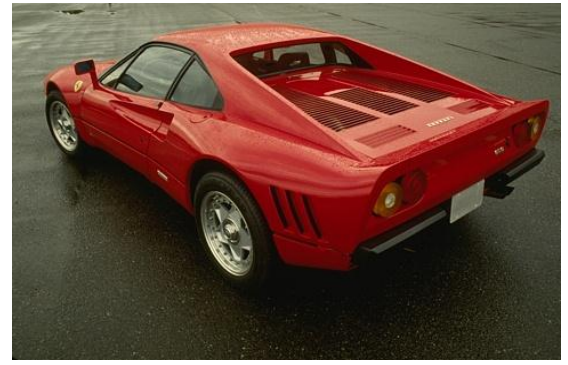

Fig 6(a)

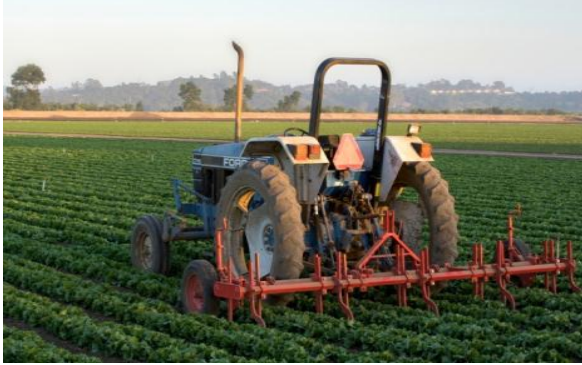

Fig 6(c)

Fig (a) and (c) Input Image

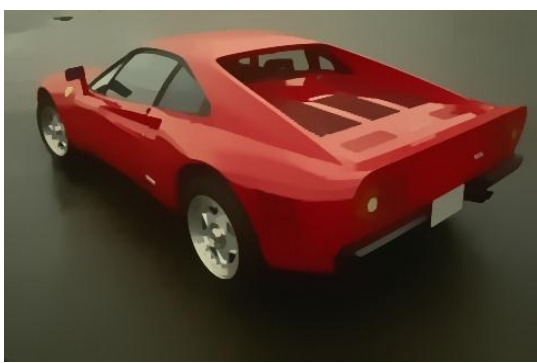

Fig 6(b)

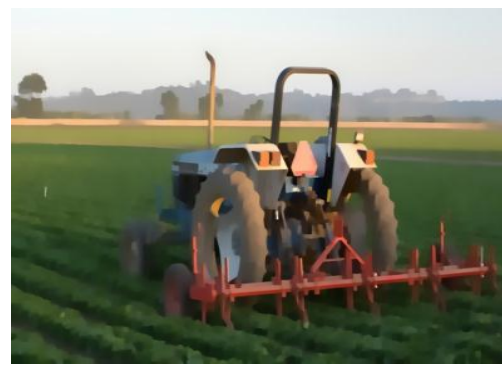

Fig 6(d)

Fig (b) and (d) output image 


\section{Conclusion}

We have presented a new system for meaningful structure extraction from texture. Our main contribution is twofold. First, we proposed novel variation measures to capture the nature of structure and texture. We have extensively evaluated these measures and conclude that they are indeed powerful to make these two types of visual information separable in many cases. Second, we fashioned a new optimization scheme to transform the original non-linear problem to a set of subproblems that are much easier to solve quickly. Several applications making use of these images and drawings were proposed. Our method does not need prior texture information. It could, thus, mistake part of structures as texture, if they are appear similar in scales.

\section{References}

[1] ARBELAEZ, P., MAIRE, M., FOWLKES, C., AND MALIK, J. 2011. Contour detection and hierarchical image segmentation. IEEE Trans. Pattern Anal. Mach. Intell. 33, 5, 898-916. ARNHEIM, R. 1956. Art and Visual Perception: A Psychology of the Creative Eye. University of California Press.

[2] AUJOL, J.-F., GILBOA, G., CHAN, T. F., AND OSHER, S. 2006. Structure-texture image decomposition - modeling, algorithms, and parameter selection. International Journal of Computer Vision

[3] AVIDAN, S., AND SHAMIR, A. 2007. Seam carving for contentaware image resizing. ACM Trans. Graph. 26, 3, 10. CANNY, J. 1986. A computational approach to edge detection. IEEE Trans. Pattern Anal. Mach. Intell. 8, 6, 679-698. DURAND, F., AND DORSEY, J. 2002. Fast bilateral filtering for the display of high-dynamic-range images. ACM Trans. Graph.

[4] Structure Extraction from Texture via Relative Total Variation • 139:9 ACM Transactions on Graphics, Vol. 31, No. 6, Article Publication Date: November 2012

[5] EFROS, A. A., AND FREEMAN, W. T. 2001. Image quilting for texture synthesis and transfer. In SIGGRAPH, 341-346. EFROS, A. A., AND LEUNG, T. K. 1999. Texture synthesis by non-parametric sampling. In ICCV, 1033-

[6] FARBMAN, Z., FATTAL, R., LISCHINSKI, D., AND SZELISKI, R. 2008. Edge-preserving decompositions for multi-scale tone and detail manipulation. ACM Trans. Graph. 27, 3.

[7] FARBMAN, Z., FATTAL, R., AND LISCHINSKI, D. 2010. Diffusion maps for edge-aware image editing. ACMTrans. Graph. 2, FATTAL, R., AGRAWALA, M., AND RUSINKIEWICZ, S. 2007. Multiscale shape and detail enhancement from multi-light image collections 